\title{
A Centralized Energy Balancing In Clustering Process for WSN
}

\author{
M. Ben Salah, A. Boulouz \\ Laboratoire des systèmes informatiques et vision LabSIV \\ Faculté des Sciences Université Ibn Zohr, Agadir, Maroc
}

\begin{abstract}
Wireless sensor networks consist of small battery powered. Devices with limited energy resources optimization of energy consumption is one of the most important challenges in WSNs due to the limited energy capacity of the network nodes. Several studies have been done in order to design energy efficient routing mechanism to increase the network lifetime. Clustering is one of the best used methods. This work proposes a centralized routing protocol used a $\mathrm{CH}$ selection by considering the remaining energy of sensor node in $\mathrm{CH}$ selecting process. Simulation results show that the proposed scheme reduces the energy consumption and prolong the network life-time of network compared to the well-known clustering algorithms LEACH.
\end{abstract}

\section{Keywords}

Wireless Sensor Networks (WSNs); Energy consumption; Life-time; Clustering; $\mathrm{CH}$; LEACH

\section{INTRODUCTION}

We ask that authors follow some simple guidelines. In essence, we ask you to make your paper look exactly like this document. The easiest way to do this is simply to download the template, and replace the content with your own materi Wireless Sensor Network (WSN) WSN consists of several sensor nodes deployed in a region to sense various types of physical informations [1]. The collected information is relayed to the base station (BS).

The major constraint of this type of network is the limitation of the energy resources of the nodes. Therefore, energy efficiency is a very crucial issue [2-3] and Energy-efficient protocols must be designed and developed to maximize network lifetime.

Many routing protocols have been proposed to improve the performance of the network, in particular the lifetime and energy consumption [2-11].

Transmission is the most energy consuming operation. Clustering is the most widely used method [5-9]. It consists in organizing the nodes as a group (cluster). Each cluster has a cluster head $(\mathrm{CH})$.. The process of cluster formation consists of two phases, $\mathrm{CH}$ election and assignment of nodes to cluster-heads. In order to minimize the transmission distance the nodes send the collected data to their own $\mathrm{CH}$ which sends it to the BS.

However, $\mathrm{CH}$ consumes much more energy than normal nodes. It is very important to take into account the residual energy of each node in the $\mathrm{CH}$ selection phase and to exchange the role of $\mathrm{CH}$ between the different nodes in order to balance the energy of the network.

In this paper, a centralized routing protocol has been proposed to improve network lifetime of WSN by balancing the energy consumption among nodes.
The rest of the paper is organized as follows. Section 2 presents the used radio energy dissipation model. Section 3 gives brief description about proposed protocol. Section 4 present simulation results and finally Section 5 concludes the paper.

\section{RADIO ENERGY MODEL DISSIPATION}

The energy required by the transmit amplifier $T_{T x}(l, d)$ to transmit 1 bit message between a transmitter and receiver over a distance $d$ is [5-9]:

$$
E_{T x}(l, d)=\left\{\begin{array}{ll}
l \times E_{e l e c}+l \times \varepsilon_{f s} \times d^{2} & \text { if } d \leq d_{0} \\
l \times E_{e l e c}+l \times \varepsilon_{m \Psi} \times d^{4} & \text { if } d \geq d_{0}
\end{array}\right\}
$$

Where $\mathrm{d}_{0}=\sqrt{\varepsilon_{f s} / \varepsilon_{m p}}$ is the threshold distance, Eelec represents the energy consumption in the electronics for sending or receiving one bit. The terms $\varepsilon_{f s} \times \mathrm{d}^{2}$ and $\varepsilon_{m p} \times \mathrm{d}^{4}$ represent respectively the amplifier energy consumptions for a short and long-distance transmissions.

\section{PROPOSED WORK}

In this work we make some assumptions:

- There are $\mathrm{N}$ sensors nodes, which are randomly deployed in square field within a MxM square region

- The nodes are The normal nodes transmit data to $\mathrm{CH}$.

- All nodes start with the same energy Eo.

- The cluster $\mathrm{CH}$ the aggregated data to the BS directly. -

- The BS position is predetermined and located outside the sensing area

- Nodes are are immobile

The base station (BS) initiates the routing process. The $\mathrm{CH}$ selection is based on residual energy level of the nodes with respect to average energy of network.

In order to balance the energy consumption among all nodes, we introduce a new cluster head selection threshold based on proposed cluster head selection probability, residual energy of a node. The improved threshold in this paper is given by (1).

$$
T(i)=\left\{\begin{array}{l}
\frac{p}{1-p^{*}\left(r \bmod \frac{1}{p}\right)} \frac{E_{a v}}{E_{i}} \text { if } \mathrm{i} \in \mathrm{G} \\
0 \text { otherwise }
\end{array}\right.
$$


In eq. $1, p$ is the optimal cluster-head probability, $r$ is the number of the current round, $G$ is set of nodes that have not been cluster heads in last $1 / p$ rounds. $E_{a v}$ is the average energy of the network.

In $\mathrm{CH}$ process each node having residual energy great or equal $\mathrm{E}_{\mathrm{av}}$ generates a uniform random number in the interval $\left[0, \mathrm{E}_{\mathrm{av} / \mathrm{Eo}}\right]$, if this number is less than the threshold $\mathrm{T}(\mathrm{s})$, then this node is candidate to become a $\mathrm{CH}$.

\section{SIMULATION RESULTS}

The propsed protocol is simulated and its performance is compared with ar clustering protocols LEACH. Performance of proposed protocol is measured in terms of network lifetime, energyefficiency, and stability period.

The used simulation are listed in Table I according to the radio basic energy dissipation model [4-6].

Table 1: Simulation Parameters

\begin{tabular}{|l|l|}
\hline \multicolumn{1}{|c|}{ Parameters } & values \\
\hline Number of nodes & 100 \\
\hline Field simulation & $100 \mathrm{~m} \mathrm{x} \mathrm{100m}$ \\
\hline Base station position & $(50,175)$ \\
\hline Initial energy (Eo) & $0.5 \mathrm{~J}$ \\
\hline Transmitter Electronics (Eelec) & $50 \mathrm{~nJ} / \mathrm{bit}$ \\
\hline Receiver Electronics $($ Eelec $)$ & $50 \mathrm{~nJ} / \mathrm{bit}$ \\
\hline Length of data packet $($ Bytes $)$ & 3000 \\
\hline Transmitter Amplifier $\left(\varepsilon_{\mathrm{fs}}\right)$ & $10 \mathrm{pJ} / \mathrm{bit} / \mathrm{m}^{2}$ \\
(If $\mathrm{d}<\mathrm{d}_{0}$ ) & \\
\hline Transmitter Amplifier $\left(\varepsilon_{\mathrm{mp}}\right)$ & $0.0013 \mathrm{pJ} / \mathrm{bit} / \mathrm{m}^{4}$ \\
(If $\left.\mathrm{d}>\mathrm{d}_{0}\right)$ & \\
\hline
\end{tabular}

Figure 1 show clearly that the proposed algorithm extends network lifetime compared with LEACH. The network becomes completely off after the round 1605 and 2500 for both protocols LEACH and proposed algorithm. Also the proposed algorithm enhance the stability period (also known as a number of rounds after which death of first sensor node occurs). The results are summarized in table 2 .

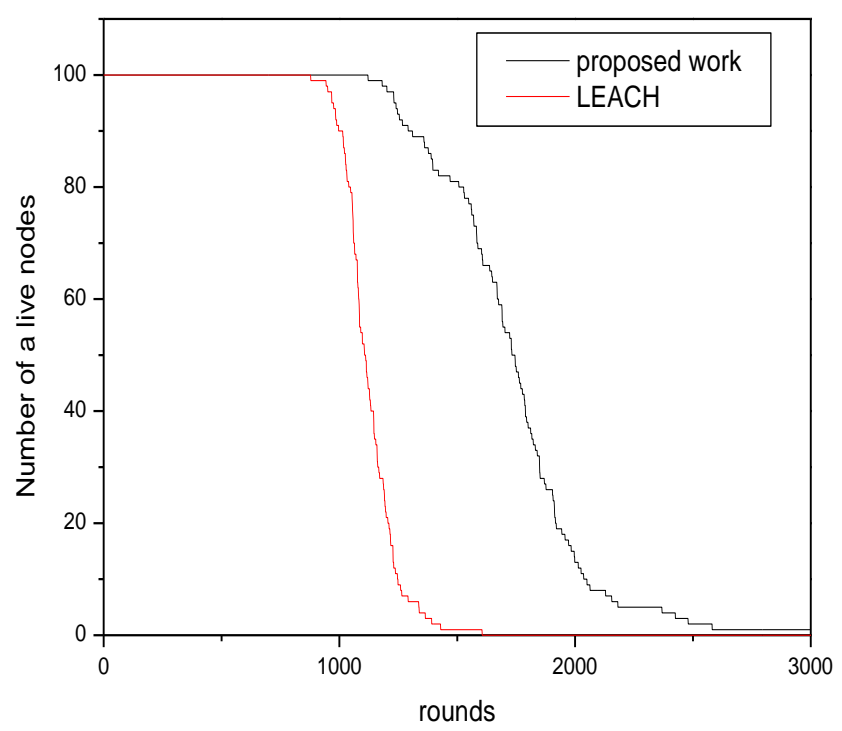

Fig. 1: Simulation result of network lifetime.

Table 2: Comparison of Network Parameters

\begin{tabular}{|c|c|c|}
\hline & LEACH & proposed \\
\hline First node die & 879 & 1120 \\
\hline Last node die & 1605 & 2500 \\
\hline
\end{tabular}

The dissipated energy of the entire network for the proposed protocol compared to LEACH protocol is shown in Fig. 2. It is shown clearly that the proposed protocol reduce average dissipated energy than LEACH.

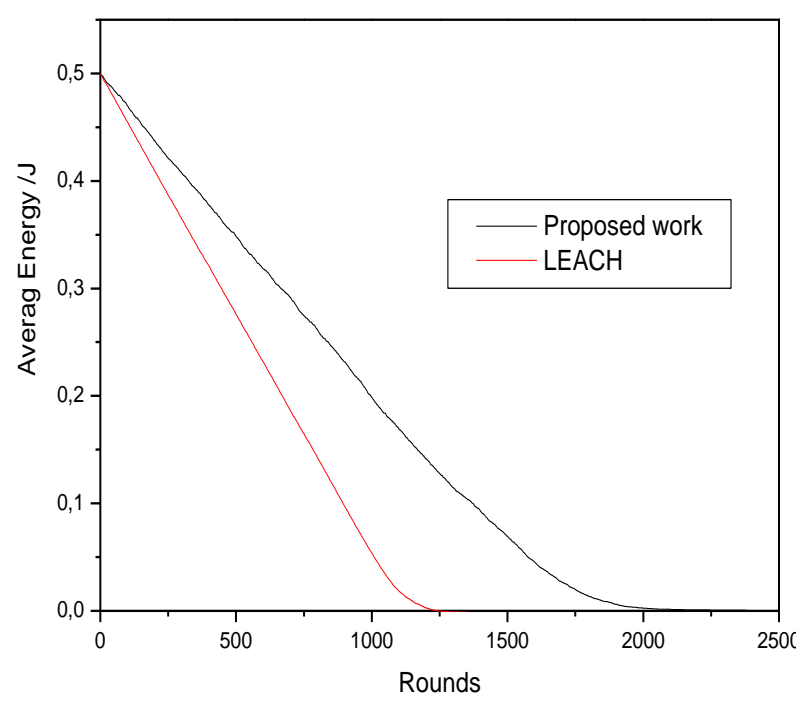

Fig. 2: The energy consumption

\section{CONCLUSION}

This paper proposed a new clustering scheme to improve network lifetime and stability period for WSN. A new centralized $\mathrm{CH}$ algorithm based on the nodes residual energy and the average energy of the entire network.

The simulation results showed that the proposed protocol is more energy efficient and enhanced the network lifetime and period stability as compared to LEACH. 


\section{REFERENCES}

[1] Y. He, W. S. Yoon and J. H. Kim. "Multi-levelClustering Architecture for Wireless Sensor Networks". Information Technology Journal, Vol. 5, No. 1, 2006, pp. 188-191

[2] C. Alippi, G . Anastasi, MD Francesco, Roveri M, " An adaptive sampling algorithm for effective energy Management in wireless sensor networks with energyhungry sensors", IEEE Trans Instrum Meas 2010;59(2):335-44.

[3] US Sutar, SK Bodhe, 'Energy efficient topology control algorithm for multi-hop ad-hoc wireless sensor network', In: Proc. 3rd IEEE international conference on computer science and information technology (ICCSIT), Chengdu, China; July 2010

[4] Hai-Ying Zhou, Dan-Yan Luo, Yan Gao, De-Cheng Zuo, "'Modeling of Node Energy Consumption for Wireless Sensor Networks", Wireless Sensor Network, 2011,3, $18-23$

[5] W. Heinzelman, A. Chandrakasan, and H. Balakrishnan, "Energy-efficient routing protocols for wireless microsensor networks," in Proc.33rd Hawaii Int. Conf. System Sciences (HICSS), Maui, HI, Jan. 2000.
[6] Handy MJ, Haase M, Timmermann D. Low energy adaptive clustering hierarchy with deterministic clusterhead selection. In: Proc. 4th IEEE conference on mobile and wireless communication networks; 2002. p. 368-72.

[7] S. Ali Md, D. Tanay, B. Rahul, "ALEACH advanced LEACH routing protocol for wireless microsensor networks", In: Proc. ICECE 2008, vols. 1 and 2; 2008. p. 909-14.

[8] A.Wang, D. Yang, D. Sun, "A clustering algorithm based on energy information and cluster heads expectation for wireless sensor networks". Computers and Electrical Engineering 38 (2012) 662-671

[9] W.R.Heinzelman, A.P.Chandrakasan, H.Balakrishnan, "An application- specific protocol architecture for wireless microsensor networks", IEEETransactions on Wireless Communications (4) (2002) 660-670.

[10] Rohit D. Gawade and S. L. Nalbalwar," A Centralized Energy Efficient Distance Based Routing Protocol for Wireless Sensor Networks", Journal of Sensors, 2016 (2016).

[11] Prabhudutta Mohanty, Manas Ranjan Kabat, "Energy efficient structure-free data aggregation and delivery in WSN". Egyptian Informatics Journal (2016) 17, 273-284 